\title{
Clinicopathologic, surgical characteristics and survival outcomes of rectal gastrointestinal stromal tumors
}

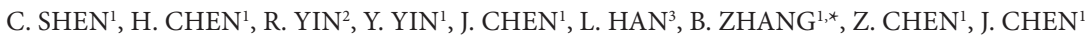 \\ ${ }^{1}$ Department of Gastrointestinal Surgery, West China Hospital, Sichuan University, Chengdu 610041, Sichuan, China; ${ }^{2}$ Department of Pathol- \\ ogy, West China Hospital, Sichuan University, Chengdu 610041, Sichuan, China; ${ }^{3}$ Intensive Care Unit, West China Hospital, Sichuan University, \\ Chengdu 610041, China
}

*Correspondence: hxwcwk@126.com

Received October 8, 2014 / Accepted January 7, 2015

Rectal gastrointestinal stromal tumors (GISTs) are rare, and limited information is available concerning their management and long-term outcomes. We retrospectively evaluated the clinicopathological characteristics, surgical management, and long-term outcomes of rectal GISTs from a single institution.

All surgically treated patients with rectal GISTS at the Department of General Surgery, West China Hospital, Sichuan University were identified between January 2005 and May 2014. The overall survival (OS) and disease-free survival (DFS) were assessed by the Kaplan-Meier method.

Forty-five patients with rectal GISTs (33 males and 12 females) were identified. Patients presented with rectal bleeding $(n=13 ; 28.9 \%)$ and altered bowel habits $(n=11 ; 24.4 \%)$. The cohort study of 45 patients included 4 very low-risk, 10 lowrisk, 1 intermediate-risk, and 30 high-risk patients. A total of 21, 13, and 11 patients underwent local resection (Group 1), abdominoperineal resection (Group 2), and super-low or low anterior resection (Group 3), respectively. Group 1 had a smaller tumor sizes and shorter distances from the anal verge compared with the other groups $(P<0.05)$. The one-, three-, and fiveyear DFS rates for the entire cohort study were $90.4 \%, 69.3 \%$, and $57.0 \%$, respectively. High National Institutes of Health $(\mathrm{NIH})$ risk categories $(\mathrm{HR}=1.62)$ were associated with low DFS rates $(\mathrm{P}=0.035)$. The DFS was significantly improved by imatinib mesylate $(\mathrm{IM})$ adjuvant therapy in the high-risk subgroup $(\mathrm{P}=0.001)$.

The type of surgery should be chosen based on the location and size of the rectal GISTs. Adjuvant IM therapy was associated with improved DFS in patients with high-risk tumors, and classification was strongly associated with the patient outcome.

Key words: gastrointestinal stromal tumors, rectum, clinicopathologic

Gastrointestinal stromal tumors (GISTs) were misdiagnosed as smooth muscle or neurogenic tumors for decades. Mazur and Clark re-evaluated the histogenesis of GISTs in 1983, and subsequent research has confirmed that GISTs are the most common mesenchymal tumors of the gastrointestinal tract; these tumors are believed to arise from the interstitial cells of Cajal or their precursors [1-3]. The annual incidence of GISTs has been estimated in the range $10 / 10^{6}$ to $15 / 10^{6}$ [4-5]. GISTs usually originate from the stomach (50\% to $60 \%$ ) and small intestines (20\% to $30 \%$ ); approximately only $5 \%$ of GISTS originate from the rectum, which account for $0.1 \%$ of all rectal tumors [6-7].To date, surgery with histologically negative margins is the cornerstone of treatment for primary resectable GISTS [8]. However, rectal GISTs with R0 resec- tion are challenging for surgeons because of the involvement of adjacent structures, such as the prostate in males and the vagina in females [9]. Therefore, multivisceral resections may be needed for complete surgical resection of rectal GISTs. Surgeons currently select the surgical procedure based on the tumor location, but no standardized guidelines for selection are available.

The available literature on rectal GISTs is limited to date; most studies on rectal GISTs have small sample sizes [10-11]. In the current study, we aimed to evaluate the clinicopathological characteristics, surgical management, and long-term outcomes of rectal GISTs of patients from a single medical institution. The prognostic factors associated with disease-free survival (DFS) were determined. 


\section{Patients and methods}

Patient selection. Medical charts of patients who received a histological diagnosis of rectal GISTs and who underwent surgical treatment between January 2005 and May 2014 were retrospectively analyzed. We reviewed the medical records to obtain data on the demographic and clinicopathological characteristics, surgical interventions, medication, therapy, and recurrence/metastasis events. Preoperative abdominal CT scans, colonoscopy, and trans-rectal sonography were routinely performed to evaluate the tumors. All pathological slides were reviewed by a senior pathologist to confirm the previous diagnosis. Patients with other malignant tumors at diagnosis or with incomplete medical records were excluded from the study.

Surgery and medication management. Surgical procedures were considered to achieve $\mathrm{R} 0$ resection (i.e., the absence of microscopically residual tumor and rupture of tumor) as much as possible. Patients were grouped based on the type of operation performed, as follows: trans-abdominal or anal local resection (Group 1), abdominoperineal resection (Group 2), and super-low or low anterior resection (Group 3). Patients underwent one of these three surgical procedures, and the selection was mainly based on the tumor size and location. The surgical margins were pathologically confirmed as negative, and the digestive tract was reconstructed. Tumor risk categories were evaluated according to the modified National Institutes of Health (NIH) classification [12]. The indicated preoperative and adjuvant treatments with imatinib mesylate (IM) for intermediate- and high-risk patients were administered by the attending clinicians with the patient's consent. The recommended IM dosage was $400 \mathrm{mg} / \mathrm{d}$. Patients that received preoperative IM therapy underwent a trans-rectal biopsy to confirm the diagnosis, according to the National Comprehensive Cancer Network guidelines [13]. All patients signed informed consent before receiving any treatment.

Statistical analysis and follow-up. Median values were used to describe the continuous data. Measurement data was expressed as mean \pm standard deviation. Categorical data from different groups was compared using the $\chi^{2}$ test or Fisher's exact test. One-way ANOVA was used to compare continuous data. DFS was calculated from the time of operation to either biopsy-proven or radiologic evidence of recurrent or metastatic disease. The overall survival (OS) was defined as the period from surgery to the patient's death or until the last follow-up. Cumulative event rates were estimated by the
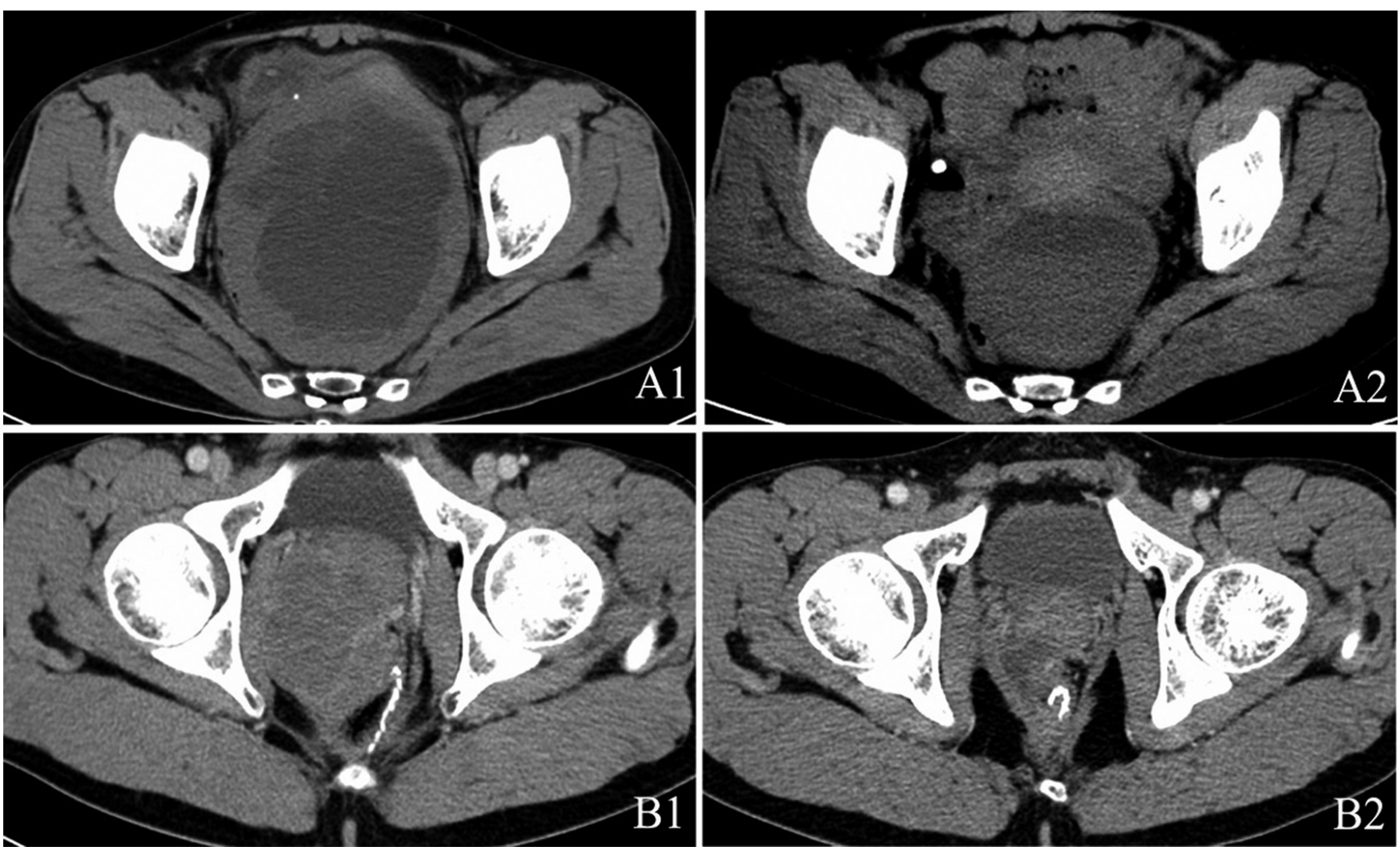

Figure 1. Representatives of preoperative therapy with imatinib mesylate. A1: CT scan shows a mass with approximately $12 \mathrm{~cm} \times 11 \mathrm{~cm}$ in size located in the pelvis. A2: Tumor had shrunk to the size of $6.4 \mathrm{~cm} \times 5.9 \mathrm{~cm}$ after 4 months of preoperative IM therapy with a dosage of $400 \mathrm{mg} / \mathrm{d}$. B1: A patient with anastomotic recurrence of tumor size $7.8 \mathrm{~cm} \times 6.5 \mathrm{~cm}$ (The tumor was excised 8 years ago). B2: Tumor size reduced to $2.7 \mathrm{~cm} \times 2.6 \mathrm{~cm}$ with a duration of 6 months of preoperative IM. Eventually, he received reoperation. 
Kaplan-Meier method and compared by the log-rank test. The Cox proportional hazards regression model were used to estimate the hazard ratios for DFS. $P<0.05$ was considered statistically significant. All data analyses were performed using the SPSS version 17.0 statistical software package for Microsoft Windows. Follow-up was performed through regular outpatient visits and telephone calls to the patient after the patient was discharged from the hospital (at intervals of 3 months to 6 months). Abdominal and/or pelvic enhanced CT was routinely performed. The median follow-up of this series was

Table 1. The demographic and tumor characteristics of patients with rectal GISTs

\begin{tabular}{|c|c|c|}
\hline Variables & $\begin{array}{l}\text { No. of Patients } \\
\qquad(\mathrm{n}=45)\end{array}$ & $\begin{array}{c}\text { Percentage } \\
(\%)\end{array}$ \\
\hline \multicolumn{3}{|l|}{ Age (years) } \\
\hline$\leq 60$ & 30 & 66.7 \\
\hline$>60$ & 15 & 33.3 \\
\hline \multicolumn{3}{|l|}{ Gender } \\
\hline Male & 33 & 73.3 \\
\hline Female & 12 & 26.7 \\
\hline \multicolumn{3}{|l|}{ Clinical presentation } \\
\hline Rectal pain & 8 & 17.8 \\
\hline Rectal bleeding & 13 & 28.9 \\
\hline Difficult defecation & 5 & 11.1 \\
\hline Change in bowel habits & 11 & 24.4 \\
\hline Urinary complaints & 3 & 6.7 \\
\hline Others $^{*}$ & 5 & 11.1 \\
\hline \multicolumn{3}{|l|}{ Tumor size $(\mathrm{cm})$} \\
\hline$\leq 5$ & 25 & 55.6 \\
\hline$>5$ & 20 & 44.4 \\
\hline \multicolumn{3}{|l|}{ Distance from anal verge $(\mathrm{cm})$} \\
\hline$\leq 5$ & 28 & 62.2 \\
\hline $6-10$ & 11 & 24.4 \\
\hline$>10$ & 6 & 13.3 \\
\hline \multicolumn{3}{|l|}{ Mitotic index (50HPF) } \\
\hline$\leq 5$ & 24 & 53.3 \\
\hline $6-10$ & 16 & 35.6 \\
\hline$>10$ & 5 & 11.1 \\
\hline \multicolumn{3}{|l|}{ NIH risk categories } \\
\hline Very low & 4 & 8.9 \\
\hline Low & 10 & 22.2 \\
\hline Intermediate & 1 & 2.2 \\
\hline High & 30 & 66.7 \\
\hline Preoperative IM therapy & 3 & 6.7 \\
\hline Adjuvant IM therapy & 13 & 28.9 \\
\hline Accompanied with rectal polyps & 3 & 6.7 \\
\hline Liver metastasis at diagnosis & 1 & 2.2 \\
\hline Reoperation for recurrent patients & 6 & 13.3 \\
\hline Median follow-up (range, months) & $38(3-115)$ & - \\
\hline Hospital stay (days, mean \pm SD) & $14.0 \pm 4.1$ & - \\
\hline
\end{tabular}

${ }^{\star}$ Others included incidentally found and palpable mass.

NIH: National Institutes of Health.

HPF: High power field.

IM: Imatinib mesylate.

SD: Standard deviation
38 months (in the range 3 months to 115 months). The censor date of the follow-up was August 2014.

\section{Results}

Patient demographics and tumor characteristics. We included 45 rectal GISTs patients, among which 33 were males and 12 were females, with a median age of 55 years old $(\leq 60$ years old in 30 cases and $>60$ years old in 15 cases). Patients with rectal GISTS presented several symptoms, including the following: rectal pain $(n=8 ; 17.8 \%)$, rectal bleeding $(n=13$; $28.9 \%)$, difficulty with defecation $(n=5 ; 11.1 \%)$, change in bowel habits $(n=11 ; 24.4 \%)$, urinary complaints $(n=3 ; 6.7 \%)$, and others $(n=5 ; 11.1 \%$, including incidentally found and palpable mass). The tumor sizes varied from $1 \mathrm{~cm}$ to $13 \mathrm{~cm}$, with a median size of $5 \mathrm{~cm}$ (25 patients with $\leq 5 \mathrm{~cm}$ and 20 patients with $>5 \mathrm{~cm}$ ). Tumor sites were mainly located in the lower two-thirds of the rectum. The cohort study of 45 patients included 4 very low-risk, 10 low-risk, 1 intermediate-risk, and 30 high-risk patients. A total of 8 gene mutations were available (6 mutations in KIT exon 11, 1 mutation in KIT exon 9, and 1 mutation with wild type). Genetic data for the remaining cases were unavailable because of the patient's refusal to undergo genotyping mainly due to economic reasons. Three patients notably showed rectal polyps. Three other patients were treated with preoperative IM at a standard dosage of $400 \mathrm{mg} / \mathrm{d}$, and medication continued for 5, 10, or 12 months. Partial response was obtained from these three patients. One patient with local tumor relapse received preoperative IM therapy and underwent reoperation (Figure 1). Adjuvant IM dose of $400 \mathrm{mg} / \mathrm{d}$ was administered to 13 patients $(28.9 \%)$ for a median time of 18 months (in the range 3 months to 46 months). A number of high-risk patients refused IM therapy mainly due to economic reasons. In one of these patients, the IM dosage was reduced to $300 \mathrm{mg} / \mathrm{d}$ because the patient presented with abnormal liver function, severe vomiting, and nausea, as shown in Table 1.

Surgical outcome. The clinicopathological features of the three types of surgical procedures are listed in Table 2. All patients in this cohort received surgical treatment. Transabdominal or anal local resection (Group 1) was performed in 21 patients (46.7\%). Abdominoperineal resection (Group 2) was conducted in 13 cases $(28.9 \%)$, whereas 11 patients (24.4\%) underwent super-low or low anterior resection (Group 3). Compared with Group 1, Group 2 patients stayed in the hospital longer $(12.1 \pm 3.2 \mathrm{~d}$ vs. $17.1 \pm 3.8 \mathrm{~d}, \mathrm{P}=0.001)$, had larger tumor sizes $(3.4 \pm 1.8 \mathrm{~cm}$ vs. $7.3 \pm 3.3 \mathrm{~cm}$, $\mathrm{P}=0.000)$, and had tumors located at greater distances from the anal verge $(4.3 \pm 2.1 \mathrm{~cm}$ vs. $6.6 \pm 2.5 \mathrm{~cm}, \mathrm{P}=0.026)$. Group 3 patients showed larger tumor sizes and had tumors located at greater distances from the anal verge $(\mathrm{P}<0.05)$ than Group 1 patients. Group 2 comprised a higher number of intermediate- and high-risk patients than Group $1(\mathrm{P}=0.003)$. Two patients in Group 2 had multivisceral resection (one had prostatectomy and the other one had uterectomy). Four patients $(8.9 \%)$ experienced postoperative complications, 
Table 2. The tumor characteristics and surgical outcomes of three operative interventions

\begin{tabular}{|c|c|c|c|}
\hline Variables & Group $1(n=21)$ & Group $2(n=13)$ & Group $3(n=11)$ \\
\hline Gender (M/F) & $17 / 4$ & $9 / 4$ & $7 / 4$ \\
\hline Age (years, mean $\pm \mathrm{SD}$ ) & $53.5 \pm 10.0$ & $58.6 \pm 10.3$ & $57.4 \pm 13.3$ \\
\hline Hospital stay (days, mean \pm SD) & $12.1 \pm 3.2^{\mathrm{a}}$ & $17.1 \pm 3.8$ & $14.6 \pm 4.0$ \\
\hline Tumor size (cm, mean $\pm \mathrm{SD})$ & $3.4 \pm 1.8^{\mathrm{a}}$ & $7.3 \pm 3.3$ & $6.0 \pm 2.3^{\mathrm{c}}$ \\
\hline Distance from anal verge $(\mathrm{cm}$, mean $\pm S D)$ & $4.3 \pm 2.1^{\mathrm{a}}$ & $6.6 \pm 2.5^{\mathrm{b}}$ & $9.1 \pm 4.1^{\mathrm{c}}$ \\
\hline \multicolumn{4}{|l|}{ NIH risk categories } \\
\hline Very low and low (\%) & $11(52.4)$ & $0(0.0)$ & $3(27.3)$ \\
\hline Intermediate and high (\%) & $10(47.6)$ & $13(100.0)$ & $8(72.7)$ \\
\hline \multicolumn{4}{|l|}{ Surgical margins status } \\
\hline R0 (\%) & $21(100.0)$ & $12(92.3)$ & $10(90.9)$ \\
\hline $\mathrm{R} 1(\%)$ & $0(0.0)$ & $1(7.7)$ & $1(9.1)$ \\
\hline Intestinal obstruction (\%) & $0(0.0)$ & $1(7.7)$ & $0(0.0)$ \\
\hline Abdominal infection/abscess (\%) & $0(0.0)$ & $0(0.0)$ & $1(9.1)$ \\
\hline Wound infection/ dehiscence (\%) & $0(0.0)$ & $1(7.7)$ & $0(0.0)$ \\
\hline Anastomotic fistula (\%) & $0(0.0)$ & $0(0.0)$ & $0(0.0)$ \\
\hline Rectal bleeding (\%) & $1(4.8)$ & $0(0.0)$ & $0(0.0)$ \\
\hline Multivisceral resection (\%) & $0(0.0)$ & $2(15.4)$ & $0(0.0)$ \\
\hline Tumor recurrence/metastasis (\%) & $10(47.6)$ & $5(38.5)$ & $3(27.3)$ \\
\hline Overall survival (months ) & $61.2 \pm 36.5$ & $37.7 \pm 27.4$ & $54.9 \pm 33.8$ \\
\hline Disease free survival (months) & $47.6 \pm 38.5$ & $32.3 \pm 25.6$ & $51.3 \pm 32.5$ \\
\hline
\end{tabular}

a: comparison between Group land 2, $P<0.05$.

b: comparison between Group 2 and Group 3, $P<0.05$.

c: comparison Group 1 and Group 3, $P<0.05$.

NIH: National Institutes of Health.

SD: Standard deviation.

such as intestinal obstruction ( $n=1)$, abdominal infection/ abscess $(n=1)$, wound infection/dehiscence $(n=1)$, and rectal bleeding $(n=1)$. Only one patient (with anastomotic fistula) required reoperation. The three groups were not significantly different in terms of gender, age, surgical margin status, OS time, and DFS time of patients. However, a trend for better OS was achieved in Group 1 compared with Group $2(\mathrm{P}=0.053)$.

Tumor immunohistochemical features. Immunohistochemical investigation revealed that 1 tumor (2.2\%) and 39 tumors $(86.7 \%)$ stained focal positive and positive for CD117, respectively. A total of 36 tumors (80.0\%) stained positive for CD34, 5 tumors (11.1\%) stained positive for S-100 proteins, 6 tumors (13.3\%) stained positive for smooth muscle actin (SMA), and 2 tumors (4.4\%) stained positive for desmin. The Ki-67 labeling index was $\leq 5 \%$ in 20 patients (44.4\%), $6 \%$ to $10 \%$ in 19 patients $(42.2 \%$ ), and $>10 \%$ in 6 patients (13.3\%). The mean Ki-67 labeling index was 7.6\%. The tumor recurrence/metastasis was not statistically significant for the tumors that were positive for CD117, CD34, S-100, SMA, or desmin and the unstained tumors (Table 3). Most patients had a mitotic count of $\leq 5$ mitosis events per 50 high-power fields $(n=24 ; 53.3 \%)$. As shown in Figure 2.

Survival analysis. After a median follow-up period of 38 months (in the range 3 months to 115 months), the median DFS was 22 months (in the range 5 months to 96 months). However, the median OS was not reached. After the last follow-up, 19 patients showed tumor recurrence or metastasis. Among these 19 patients, 6 patients (13.3\%) underwent surgical resection again. One case had postoperative thigh root

Table 3. Tumor immunohistochemical features of rectal GISTs $(n=45)$

\begin{tabular}{|c|c|c|c|c|}
\hline Variables & Case No. & $\begin{array}{c}\text { Recurrence/ } \\
\text { metastasis }\end{array}$ & $\begin{array}{c}\text { No recurrence/ } \\
\text { metastasis }\end{array}$ & $\mathrm{P}$ value \\
\hline CD117 (\%) & & & & 1.000 \\
\hline- & $5(11.1)$ & $2(40.0)$ & $3(60.0)$ & \\
\hline+ & 40 (88.9) & $17(42.5)$ & $23(57.5)$ & \\
\hline CD34 (\%) & & & & 0.264 \\
\hline - & $9(20.0)$ & $2(22.2)$ & $7(77.8)$ & \\
\hline+ & $36(80.0)$ & $17(47.2)$ & $19(52.8)$ & \\
\hline S-100 (\%) & & & & 1.000 \\
\hline- & $40(88.9)$ & $17(42.5)$ & $23(57.5)$ & \\
\hline+ & $5(11.1)$ & $2(40.0)$ & $3(60.0)$ & \\
\hline SMA (\%) & & & & 0.686 \\
\hline- & $39(13.3)$ & $16(41.0)$ & $23(59.0)$ & \\
\hline+ & $6(86.7)$ & $3(50.0)$ & $3(50.0)$ & \\
\hline Desmin (\%) & & & & 1.000 \\
\hline - & $43(95.6)$ & $18(41.9)$ & $25(58.1)$ & \\
\hline+ & $2(4.4)$ & $1(50.0)$ & $1(50.0)$ & \\
\hline Ki-67 index (\%) & & & & 0.178 \\
\hline$\leq 5 \%$ & $20(44.4)$ & $6(30.0)$ & $14(70.0)$ & \\
\hline $6-10 \%$ & $19(42.2)$ & $10(52.6)$ & $9(47.4)$ & \\
\hline$>10 \%$ & $6(13.3)$ & $3(50)$ & $3(50)$ & \\
\hline
\end{tabular}



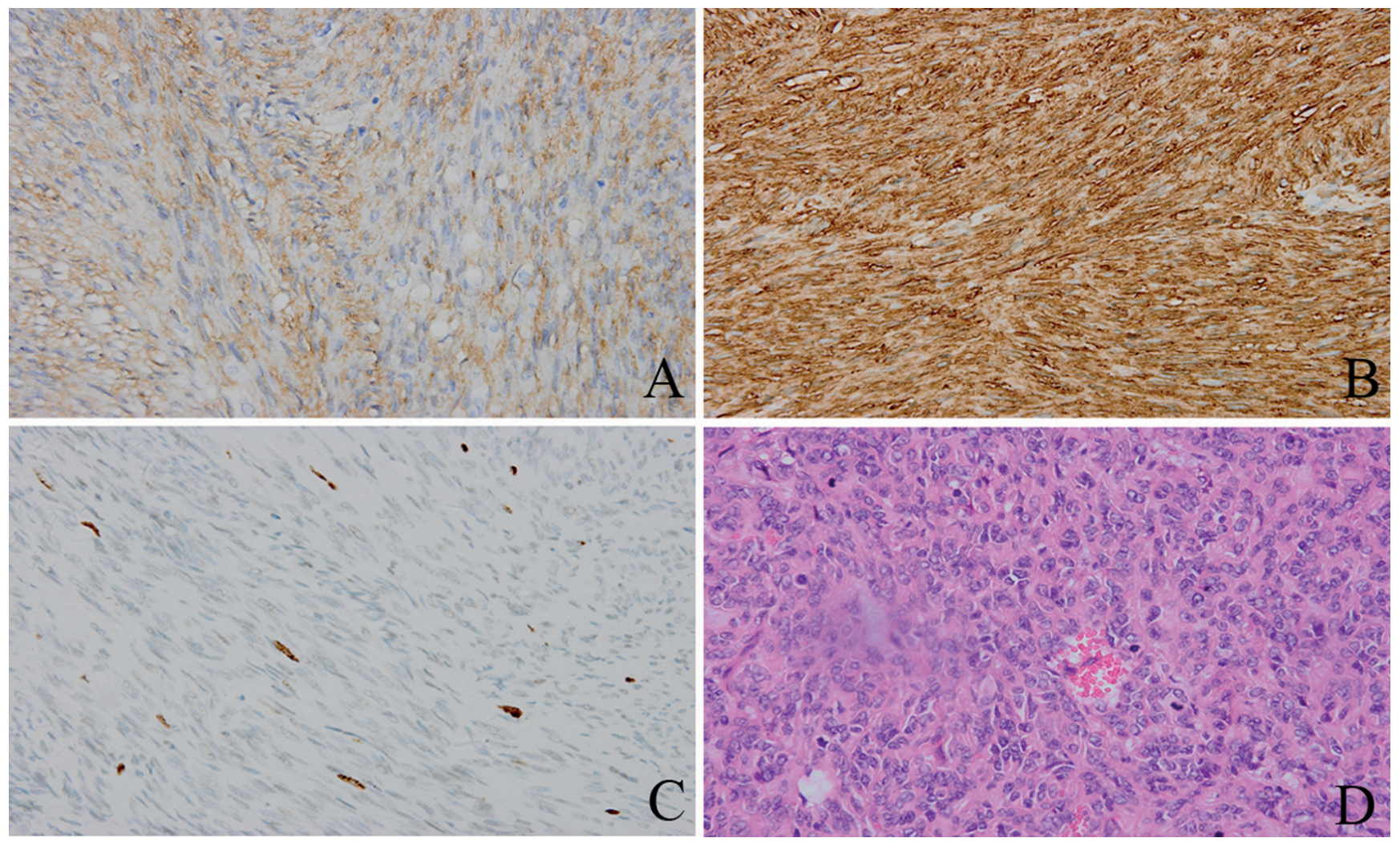

Figure 2. Tumor immunohistochemical features of rectal GISTs patients. The lesion was stained positive for CD117 (A, $\times 400)$ and CD34 (B, $\times 400)$. The Ki-67 labeling index was approximately to $8 \%(C, \times 400)$. Mitotic count of $>10$ mitosis events per 50 high-power fields were observed $(\mathrm{D}, \times 400)$
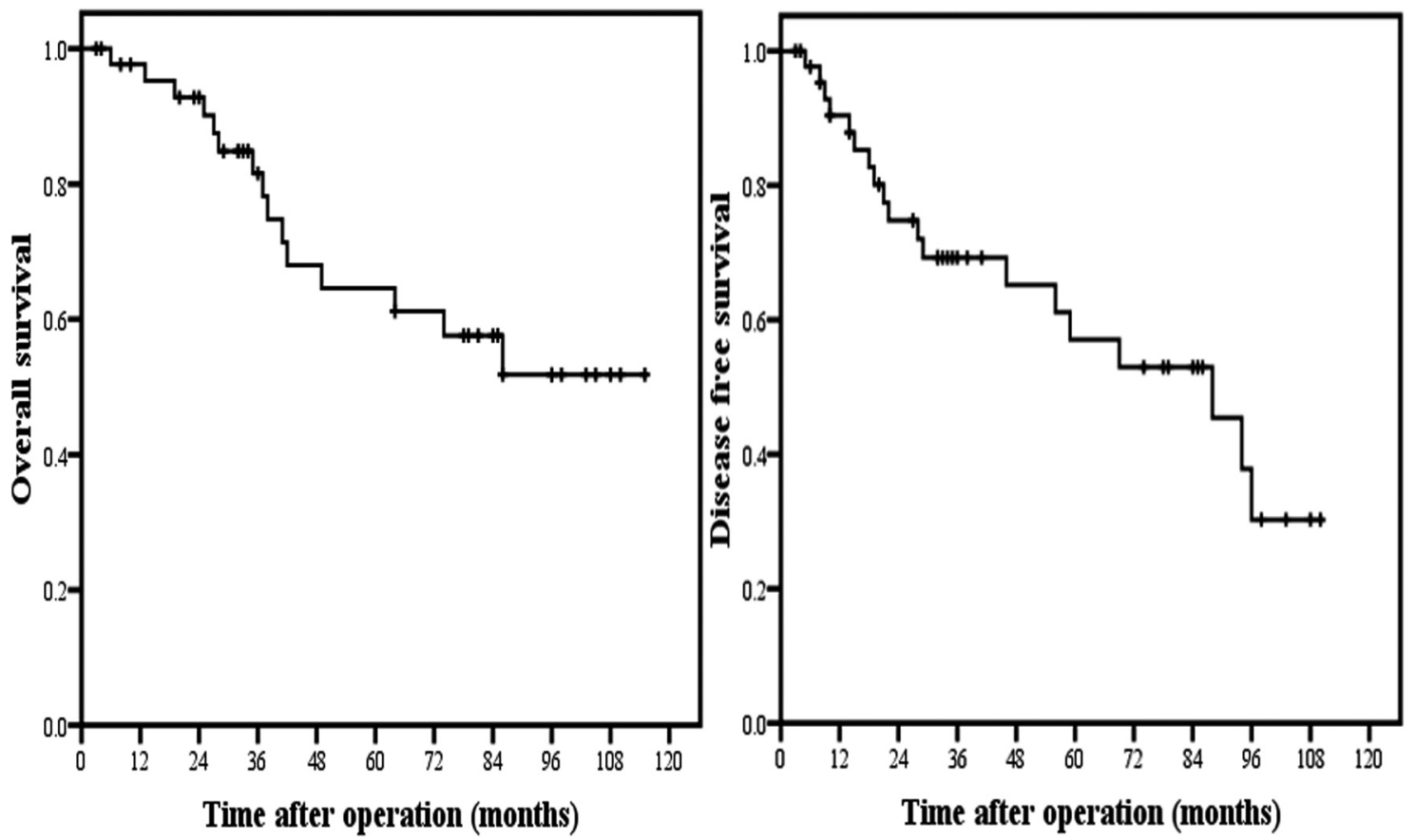

Figure 3. Kaplan-Meier curves for OS and DFS of rectal GISTs patients with surgically treated. The 1-, 3- and 5-year DFS for the entire cohort were $90.4 \%, 69.3 \%$ and $57.0 \%$, respectively. OS for the entire population were $97.7 \%$ at 1 year, $81.6 \%$ at 3 years, and $64.6 \%$ at 5 years $(n=45)$. 
metastasis after eight years. The one-, three- and five-year DFS for the entire cohort were $90.4 \%, 69.3 \%$, and $57.0 \%$, respectively. The OS rate for the entire population was $97.7 \%$ after one year, $81.6 \%$ after three years, and $64.6 \%$ after five years (Figure 3). The important predictors of DFS, as identified by univariate analysis, were age, NIH risk category, and mitotic index (Table 4). However, the multivariate Cox regression model showed that only the NIH risk categories were the independent prognostic factor for DFS (hazard ratio, 1.62; 95\% confidence interval, 1.034 to $2.551 ; \mathrm{P}=0.035)$. In addition, the high-risk subgroup of patients who received IM adjuvant treatment $(n=13)$ had significantly longer median DFS than those that were not subjected to IM treatment $(n=17 ; 34$ months vs. 20 months, $\mathrm{P}=0.001$ ).

\section{Discussion}

Limited data are available on the clinical presentation, surgical management, and survival of rectal GISTs. However, the number of studies focusing these rare tumors is growing. In the current study, we defined the presentation of patients with rectal GISTs and explored the prognostic factors associated with DFS. Our findings demonstrated that adjuvant IM therapy was associated with improved DFS in patients with high-risk tumors. The NIH classification was relatively strongly associated with patient outcomes.

Rectal GISTs occurred mainly in adults (median age of 59 years old), which is in line with the data in previous studies [14]. Our cohort of patients was predominantly male (73.3\%), which is similar to data in other studies $[9,10]$. The tumor size of rectal GISTs patients was small with a median size of $5 \mathrm{~cm}$, which was in agreement with the data obtained by Jakob et al. [15]. Nonspecific clinical presentations for rectal GISTs could be used, especially when small tumors are involved. Consistent with previous reports, patients with rectal GISTS in the current study generally presented with gastrointestinal bleeding and altered bowel habits [8]. However, some patients were asymptomatic, including those with palpable masses that were incidentally found. Therefore, surgeons and endoscopy experts must be fully acquainted with this disease. In addition, we found that most of the tumor sites were located in the lower two-thirds of rectum, as previously reported [16]. High-risk patients with rectal GISTs account for most cases (66.7\%) in the present study. This result agrees with most of the results of previous studies [9,15], except those of Zhou [14].

Immunohistochemical investigation is critical for the diagnosis of GISTs. CD117 is a TKI receptor that is the most important molecular diagnostic marker for GISTS. This marker is expressed in more than $90 \%$ of stromal tumors. Tielen et al. [10] reported that 32 patients with rectal GISTs had $88 \%$ CD117-positive expression rate. Furthermore, high positive expression of CD117 (93\%) for this type of lesion was reported [14]. Consistent with previous findings, we found that $86.7 \%$ of patients in this series were positive for CD-117. CD117 positivity is not the gold standard for the diagnosis of GISTS, but it is a major defining characteristic of GISTs. The Ki-67 protein is a cellular marker for proliferation and is expressed in the nuclei of cells in the late $G_{1}, S, G_{2}$, and $M$ phases of the cell cycle. Approximately $44.4 \%$ patients in our cohort study had a Ki-67 labeling index of $\leq 5 \%$, whereas $42.2 \%$ cases had $6 \%$ to $10 \%$ and $13.3 \%$ patients had $>10 \%$. The mean Ki-67 labeling index was $7.6 \%$, which was much higher than that of duodenal GISTs [17].

Surgical resection remains the mainstream treatment for rectal GISTs; this technique includes local resection, anterior resection, and abdominoperineal resection [10]. However, surgical resection for GISTs can be difficult and is often accompanied by considerable complications. The choice of surgical procedures for rectal GISTs is generally based on the tumor size and location. Our data showed that small GISTs located within $5 \mathrm{~cm}$ of the anal verge are ideally treated with trans-anal local resection. Limited resection is theoretically simple and feasible, but the risk of early tumor recurrence

Table 4. Univariate analysis of factors associated with disease-free survival (DFS) for rectal stromal tumors $(n=45)$

\begin{tabular}{|c|c|c|}
\hline Characteristics & $\begin{array}{l}\text { Median DFS time } \\
\quad \text { (range) }\end{array}$ & $P$ value \\
\hline Age (years) & & 0.032 \\
\hline$\leq 60(\mathrm{n}=30)$ & $33.5(3-110)$ & \\
\hline$>60(\mathrm{n}=15)$ & $33(5-96)$ & \\
\hline Gender & & 0.716 \\
\hline Male $(n=33)$ & $33(3-110)$ & \\
\hline Female $(n=12)$ & $34(4-108)$ & \\
\hline Distance from anal verge $(\mathrm{cm})$ & & 0.859 \\
\hline$\leq 5(n=28)$ & $30.5(3-110)$ & \\
\hline $6-10(n=11)$ & $32(4-94)$ & \\
\hline$>10(\mathrm{n}=6)$ & $62.5(15-85)$ & \\
\hline Types of surgery & & 0.725 \\
\hline Group $1(n=21)$ & $34(3-110)$ & \\
\hline Group $2(n=13)$ & $27(5-86)$ & \\
\hline Group $3(n=11)$ & $46(4-98)$ & \\
\hline Tumor size $(\mathrm{cm})$ & & 0.336 \\
\hline$\leq 5(n=25)$ & $34(3-110)$ & \\
\hline$>5(n=20)$ & $30(4-96)$ & \\
\hline NIH risk categories & & 0.004 \\
\hline Very low and low $(\mathrm{n}=14)$ & $59.5(3-110)$ & \\
\hline Intermediate and high $(\mathrm{n}=31)$ & $27(4-96)$ & \\
\hline Mitotic index (50HPF) & & 0.006 \\
\hline$\leq 5(\mathrm{n}=24)$ & $62.5(3-110)$ & \\
\hline $6-10(n=16)$ & $62.5(4-94)$ & \\
\hline$>10(\mathrm{n}=5)$ & $29(8-38)$ & \\
\hline IM adjuvant therapy & & 0.667 \\
\hline Yes $(n=13)$ & $35(6-96)$ & \\
\hline No $(n=32)$ & $32(3-110)$ & \\
\hline
\end{tabular}

DFS: Disease free survival. NIH: National Institutes of Health. HPF: High power field. IM: Imatinib mesylate. 
caused by incomplete excision has been noted in a previous study [18]. We observed that $47.6 \%$ of the total number of patients experienced tumor recurrence or metastasis after local resection, and this rate was higher than that of the abdominoperineal resection (38.5\%) and the super-low or low anterior resection (27.3\%). However, this difference was not statistically significant. Multivisceral resections were performed in two patients, whereas one case had R1 resection. The recurrence rate for incompletely resected GISTs notably reached up to $90 \%$ [19]. In the present study, all two patients with R1 resection showed tumor recurrence.

A previous study showed that the five-year DFS and OS for rectal GISTs were $33 \%$ and $46 \%$, respectively [ 9 ]. The five-year DFS for the current cohort was $57.0 \%$. The OS for the entire population was $64.4 \%$ after five years. The improved survival was due to the higher proportion of patients who treated with adjuvant IM therapy, as observed in the present study. Previously reported factors associated with the DFS and/or OS of GISTs include the tumor size, location, mitotic count, and NIH classification $[9,14,20]$. We found improvement in the DFS of patients showing very low- and low-risk tumors compared with in patients showing intermediate- and high-risk tumors. Previous research revealed that age may also be a prognostic factor [20]. We found that age ( $\leq 60$ years vs. $>60$ years) was associated with DFS. However, the multivariate Cox regression model excluded this variable in this series.

The drug IM played a key role in the present-day management of GISTs when used in the preoperative therapy or adjuvant treatment. Preoperative IM therapy in the present study increased the chance of radical surgery. Among the three patients with large tumors, one received surgery after preoperative IM therapy, whereas the other two obtained R0 resection. Furthermore, one patient with local tumor recurrence after preoperative IM underwent reoperation. The appropriate duration of preoperative therapy remains controversial. Generally, surgical resection is performed at 6 months to 12 months after the start of IM treatment to achieve maximum effects [21]. Previous studies showed that adjuvant IM therapy can improve the DFS and OS of GISTs patients; adjuvant IM for 3 years became the recommended standard of care for high-risk patients [22, 23]. The high-risk subgroup of patients who received IM adjuvant treatment in the present study showed significantly improved median DFS than those who did not underwent IM treatment (34 months vs. 20 months, $\mathrm{P}=0.001$ ). This result confirmed the effectiveness of IM as an adjuvant for GISTs.

In conclusion, our observations showed that the most common clinical presentation of rectal GISTs was rectal bleeding (28.9\%), followed by altered bowel habits (24.4\%). The type of surgical treatment should be chosen based on the tumor location and size. Most rectal GISTs (66.7\%) are considered to have a high risk of recurrence. The NIH risk level is the most useful independent prognostic factor for rectal GISTs. Furthermore, adjuvant IM therapy should be recommended for patients who are at high risk of recurrence.
Acknowledgements: We would like to express our warmest gratitude to Department of Gastrointestinal surgery's staffs with who generously provided assistance to collect data throughout the duration of the study.

\section{References}

[1] MAZUR MT, CLARK HB. Gastric stromal tumors: reappraisal of histogenesis. Am J Surg Pathol, 1983; 7: 507-519. http://dx.doi.org/10.1097/00000478-198309000-00001

[2] CASSIER PA, DUCIMETIERE F, LURKIN A, RANCHEREVINCE D, SCOAZEC JY, et al. A prospective epidemiological study of new incident GISTs during two consecutive years in Rhône Alpes region: incidence and molecular distribution of GIST in a European region. Br J Cancer, 2010; 103: 165-170. http://dx.doi.org/10.1038/sj.bjc.6605743

[3] KIRSCH R, GAO ZH, RIDDELL R. Gastrointestinal stromal tumors: diagnostic and practical approach to differential diagnosis. Adv Anat Pathol, 2007; 14: 261-285. http://dx.doi. org/10.1097/PAP.0b013e3180ca826a

[4] NILSSON B, BUMMING P, MEIS-KINDBLOM JM, ODEN A, DORTOK A, et al. Gastrointestinal stromal tumors: the incidence, prevalence, clinical course, and prognostication in the preimatinib mesylate era. Cancer, $2005 ; 103: 821-829$. http://dx.doi.org/10.1002/cncr.20862

[5] WOODALL CE III, BROCK GN, FAN J, BYAM JA, SCOQQINS CR, et al. An evaluation of 2537 gastrointestinal stromal tumors for a proposed clinical staging system. Arch Surg, 2009; 144: 670-678. http://dx.doi.org/10.1001/ archsurg.2009.108

[6] VAN DER ZWAN SM, DEMATTEO RP. Gastrointestinal stromal tumor: 5 years later. Cancer, 2005; 104: 1781-1788. http://dx.doi.org/10.1002/cncr.21419

[7] FLETCHER CD, BERMAN JJ, CORLESS C, GORSTEIN F, LASOTA J, et al. Diagnosis of gastrointestinal stromal tumors: A consensus approach. Hum Pathol, 2002; 33: 459-465. http:// dx.doi.org/10.1053/hupa.2002.123545

[8] CASALI PG, BLAY JY. Gastrointestinal stromal tumours: ESMO clinical practice guidelines for diagnosis, treatment and follow-up. Ann Oncol, 2010; 21: V98-102. http://dx.doi. org/10.1093/annonc/mdq208

[9] XIAO CC, ZHANG S, WANG MH, HUANG LY, WU P, et al. Clinicopathological features and prognostic factors of rectal gastrointestinal stromal tumors. J Gastrointest Surg, 2013; 17: 793-798. http://dx.doi.org/10.1007/s11605-012-2086-0

[10] TIELEN R, VERHOEF C, VAN COEVORDEN F, REYNERS AK, VAN DER GRAAF WT, et al. Surgical management of rectal gastrointestinal stromal tumors. J Surg Oncol, 2013; 107: 320-323. http://dx.doi.org/10.1002/jso.23223

[11] LIU H, YAN Z, LIAO G, YIN H. Treatment strategy of rectal Gastrointestinal stromal tumor (GIST). J Surg Oncol, 2014; 109: 708-713. http://dx.doi.org/10.1002/jso.23562

[12] JOENSUU H. Risk stratification of patients diagnosed with gastrointestinal stromal tumor. Hum Pathol, 2008; 39: 14111419. http://dx.doi.org/10.1016/j.humpath.2008.06.025

[13] DEMETRI GD, VON MEHREN M, ANTONESCU CR, DEMATTEO RP, GANJOO KN, et al. NCCN task force report: 
update on the management of patients with gastrointestinal stromal tumors. J Nat Compr Canc Netw, 2010; 8: S1-41.

[14] ZHOU Z, CHEN Z, CHEN M, WANG R, YIN Y, et al. Clinicopathologic factors predicting outcomes in patients with gastrointestinal stromal tumors of the rectum and colon. Tumor Biol, 2014; 35: 4357-4362. http://dx.doi.org/10.1007/ s13277-013-1572-7

[15] JAKOB J, MUSSI C, RONELLENFITSCH U, WARDELMANN E, NEGRI T, et al. Gastrointestinal stromal tumor of the rectum results of surgical and multimodality therapy in the era of imatinib. Ann Surg Oncol, 2013; 20: 586-592. http://dx.doi.org/10.1245/s10434-012-2647-1

[16] MACHLENKIN S, PINSK I, TULCHINSKY H, ZIV Y, SAYFAN J, et al. The effect of neoadjuvant imatinib therapy on outcome and survival after rectal gastrointestinal stromal tumour. Colorectal Dis, 2011; 13: 1110-1115. http://dx.doi. org/10.1111/j.1463-1318.2010.02442.X

[17] YANG WL, YU JR, WU YJ, ZHU KK, DING W, et al. Duodenal Gastrointestinal Stromal Tumor: Clinical, Pathologic, Immunohistochemical Characteristics, and Surgical Prognosis. J Surg Oncol, 2009; 100: 606-610. http://dx.doi. org/10.1002/jso.21378

[18] GERVAZ P, HUBER O, MOREL P. Surgical management of gastrointestinal stromal tumours. Br J Surg, 2009; 96: 567-578. http://dx.doi.org/10.1002/bjs.6601
[19] BLAY JY, BONVALOT S, CASALI P, CHOI H, DEBIECRICHTER M, et al. Consensus meeting for the management of gastrointestinal stromal tumors. Report of the GISTS Consensus Conference of 20-21 March 2004, under the auspices of ESMO. Ann Oncol, 2005; 16: 566-578. http://dx.doi. org/10.1093/annonc/mdi127.

[20] JOHNSTON FM, KNEUERTZ PJ, CAMERON JL, SANFORD D, FISHER S, et al. Presentation and Management of Gastrointestinal Stromal Tumors of the Duodenum: A MultiInstitutional Analysis. Ann Surg Oncol, 2012; 19: 3351-3360. http://dx.doi.org/10.1245/s10434-012-2551-8

[21] MUSSI C, RONELLENFITSCH U, JAKOB J, TAMBORINI E, REICHARDT P, et al. Post-imatinib surgery in advanced/ metastasis GIST: is it worthwhile in all patients? Ann oncol, 2010; 21: 403-408. http://dx.doi.org/10.1093/annonc/mdp310

[22] GRONCHI A, RAUT CP. The combination of Surgery and Imatinib in GISTS: a reality for localized tumors at high risk, an open issue for metastatic ones. Ann Surg Oncol, 2012: 19: 1051-1055. http://dx.doi.org/10.1245/s10434-011-2191-4

[23] DEMATTEO RP, BALLMAN KV, ANTONESCU CR, MAKI RG, PISTERS PW, et al. Adjuvant imatinib mesylate after resection of localised, primary gastrointestinal stromal tumour: a randomised, double-blind, placebo-controlled trial. Lancet 2009; 373: 1097-1104. http://dx.doi.org/10.1016/S0140-6736(09)60500-6 